\title{
Prognostic Value of FOXM1 in Patients with Malignant Solid Tumor: A Meta-Analysis and System Review
}

\author{
Jun Dai, ${ }^{1}$ Lili Yang, ${ }^{1}$ Jinyu Wang, ${ }^{2}$ Ying Xiao, ${ }^{1}$ and Qiurong Ruan ${ }^{1}$ \\ ${ }^{1}$ Institute of Pathology, Tongji Hospital of Tongji Medical College, Huazhong University of Science and Technology, \\ Wuhan 430030, China \\ ${ }^{2}$ Department of Pediatrics, The Second Affiliated Hospital, Zhejiang University College of Medicine, Hangzhou 310009, China
}

Correspondence should be addressed to Qiurong Ruan; ruanqiurong@sina.com

Received 17 March 2015; Accepted 23 June 2015

Academic Editor: Dinesh Kumbhare

Copyright ( $\odot 2015$ Jun Dai et al. This is an open access article distributed under the Creative Commons Attribution License, which permits unrestricted use, distribution, and reproduction in any medium, provided the original work is properly cited.

\begin{abstract}
Forkhead box M1 (FOXM1), a member of the Fox transcription factors family, was closely related with cell cycle. FOXM1 played an important role in MST and prompted a poor prognosis for MST patients. However, there were also some studies revealing no significant association between the FOXM1 expression and prognosis of patients. Therefore, we conducted meta-analysis to investigate whether the expression of FOXM1 was associated with MST prognosis. We collected 36 relevant studies through PubMed database and obtained research data of 4946 patients. Stata 12.0 was used to express the results as hazard ratio (HR) for time-to-event outcomes with $95 \%$ confidence intervals (95\% CI). It was shown that overexpression of FOXM1 was relevant to worse survival of MST patients $\left(\mathrm{HR}=1.99,95 \% \mathrm{CI}=1.79-2.21, P<0.001 ; I^{2}=26.4 \%, P_{h}=0.076\right)$. Subgroup analysis suggested that overexpression of FOXM1 in breast cancer (BC), gastric cancer (GC), hepatocellular carcinoma (HCC), pancreatic ductal adenocarcinoma (PDA), and non-small-cell lung cancer (NSCLC) all predicted a worse survival $(P<0.05)$, in addition to ovarian cancer $(\mathrm{OC})(P=0.084)$. In conclusion, our research indicated that overexpression of FOXM1 was to the disadvantage of the prognosis for majority of MST and therefore can be used as an evaluation index of prognosis.
\end{abstract}

\section{Introduction}

MST collectively referred to a large class of diseases which has a wide variety. Its morbidity and mortality vary with tumor types; however, the death toll ranks the first in the world [1].

With gradual deeper understanding of MST, molecular targeted therapy has become a major means $[2,3]$. FOXM1 is an important member of the Forkhead transcription factors family. Microarray study confirmed that the expression of FOXM1 increased in most solid tumors [4]. FOXM1, which plays key roles in cell evolution from G1 to $S$ phase and the maintenance of chromosome stability, is an important transcription factor to regulate the proliferation and apoptosis of cells [5-7]. FOXM1 can not only promote the formation of tumor by increasing cell proliferation ability, but also enhance tumor metastasis and invasion activity in advanced cancer [8-10]. Therefore, it may be said that FOXM1 fully participates in the development and progression of tumors. Numerous studies reported that FOXM1 had predictive value for MST prognosis, including BC, NSCLC, HCC, GC, and cervical cancer, [11-15]. However, there were also some studies revealing no significant association between FOXM1 and the prognosis of MST patients [16]

As factors affecting prognosis were numerous and miscellaneous, we can not simply evaluate whether one study was representative. Therefore, we conducted meta-analysis of the relationship between FOXM1 expression and prognosis in MST patients, expecting to eliminate or weaken the deficiency in studies through expanding the sample size and thereby restore the real predictive value of FOXM1.

\section{Materials and Methods}

2.1. Document Retrieval. We collected studies that have been published before January 2015 through PubMed using the following terms: "cancer" or "tumor" or "tumour" or "neoplasm") and ("FOXM1" or "FOXM1a" or "FOXM1b" or 
"FOXM1c"). We first excluded apparently unrelated studies by browsing the titles and abstracts. The full texts of all potentially eligible studies were retrieved, and their references were carefully browsed to find other studies that met the criteria. Search eligible studies by two authors independently and finally negotiate to reach consensus. The criteria were as follows: (I) research on the relationship between FOXM1 expression and the prognosis of patients in MST. (II) In the original data provided, FOXM1 expression must be divided into two grades: "positive" and "negative," regardless of FOXM1 mRNA or protein detection and the detection methods. (III) Studies must provide available data, such as HR and 95\% CI, survival curve. Similarly, we also drew up a number of exclusion criteria: (I) studies belonging to case reports, reviews, or meta-analysis, (II) authors providing only odds ratio (OR) or relative risk (RR) (we can not directly or indirectly obtain information such as $\mathrm{HR}$ and 95\% CI), and (III) research on leukemia.

2.2. Qualitative Assessment. In order to better assess the quality of the qualified studies, two independent researchers drew up the evaluation program for this study and evaluated the studies included. In short, the scoring method was based on the following five aspects: whether the diagnosis of cancer was clear; numbers of the cases; representativeness of the cases collected; judgment criteria of FOXM1; and the data sources of HR ( $95 \% \mathrm{CI})$. Each item got the maximum score of 2 points and the minimum score of 1 point (see Supplemental Table 1 in Supplementary Material available online at http://dx.doi.org/10.1155/2015/352478). The total points ranged from 5 to 10 points. Points greater than or equal to 9 points were considered to be high-quality researches.

2.3. Data Extraction. Two authors extracted information independently according to predetermined extract forms. Extract data from eligible studies included first author, year, regions, numbers of patients, tumor types, determination method of FOXM1, percentage of FOXM1 overexpression, and the sources of HR (95\% CI). If data in any of the above categories had not been reported in the original document, items were deemed as "Not Report." If the results of univariate and multivariate analysis were both reported in a study, the latter was chosen for the reason that it took the confounding factor into account and therefore it was more accurate. Taking into account the number and time of the events, the HR was the most appropriate statistic for the follow-up assessment to analyze time-to-event outcomes. For each study, the HR (95\% CI) were evaluated according to the data provided in the articles. If accurate HR (95\% CI) were given in the study, we can directly use them. In other cases, we can use Engauge Digitizer version 2.11 software to extract relevant numerical value from survival curves and calculate the HR (95\% CI) while only Kaplan-Meier survival curves were provided in the original texts $[17,18]$.

2.4. Statistical Analysis. Cochran's $Q$ and $I^{2}$ statistics were used to calculate the heterogeneity of the individual HR. As to $Q$ statistic, $P<0.05$ was considered to have statistical significance. For $I^{2}$ statistics, $I^{2}<25 \%$, no heterogeneity; $I^{2}$ $=25-50 \%$, moderate heterogeneity; and $I^{2}>50 \%$, strong heterogeneity $[19,20]$. The fixed effects model was used to combine the individual HR estimates while no significant heterogeneity was found among studies, or else, the random effects model was applied. For FOXM1 overexpression groups, HR > "1" indicated worse survival. If there was no overlap between $95 \%$ CI and "1," it would be considered that the impact of FOXM1 on survival was statistically significant. Publication bias was tested through Begg's test and Egger's test; $P<0.05$ was considered to be statistically significant. STATA 12.0 software (Stata Corporation, College Station, TX) was used to conduct all statistical analyses.

\section{Results}

3.1. Characteristics of Eligible Studies. 846 studies were preretrieved in accordance with the established search strategies. Then 171 studies that may meet the requirements were further screened out through browsing the titles and abstracts. After reading the full texts of 171 studies, 36 eligible studies were finally included in this meta-analysis according to the criteria (Figure 1). For studies included, the tumor types contained BC [11, 16, 21, 22], cervical cancer [12], colorectal cancer $[23,24]$, esophageal cancer [25], gallbladder cancer [26], GC $[14,27,28]$, glioblastoma [29], HCC [13, 30-32], laryngeal cancer [33], NSCLC [15, 34-39], malignant peripheral nerve sheath tumor (MPNST) [40], medulloblastoma [41], OC [4244], PDA [45-47], clear cell renal cell carcinoma (CCRCC) $[48,49]$, and oral cancer [35]. Characteristics of eligible studies were summarized in Table 1. Eligible studies included 4946 patients and the number of samples per study ranged from 38 to 455, with an average of 137. Eligible studies were mainly distributed in Asia (31 studies), while only 5 studies were found in non-Asia. Immunohistochemistry (IHC) was used to detect the expression of FOXM1 in vast majority of the studies. Except that one study did not mention the percentage of FOXM1 overexpression, the lowest percentage of FOXM1 overexpression was $20 \%$, while the highest was $78.7 \%$, with an average of $57.36 \%$. Among 36 studies, 16 studies provided available HR and 95\% CI, whereas 20 studies only provided survival curves. According to the qualitative assessment criteria, there were 21 studies with total scores greater than or equal to 9 points, while there was no study with less than 7 points.

3.2. Meta-Analysis. All the main results of this meta-analysis were shown in Table 2. Combine the data of 36 studies on MST to assess the cumulative survival (Figure 2). The results indicated statistically significant difference ( $\mathrm{HR}=1.99$, 95\% CI $=1.79-2.21$, and $P<0.001)$ and slight betweenresearch heterogeneity $\left(I^{2}=26.4 \%, P_{h}=0.076\right)$ between MST prognosis and FOXM1 expression.

We conducted subgroup analysis based on information provided by these studies (Table 2). We found that FOXM1 overexpression had a statistically significant effect on survival in both Asian groups and non-Asian groups, with the HR of $1.92(95 \% \mathrm{CI}=1.71-2.16)$ and $2.28(95 \% \mathrm{CI}=1.81-2.89)$, 


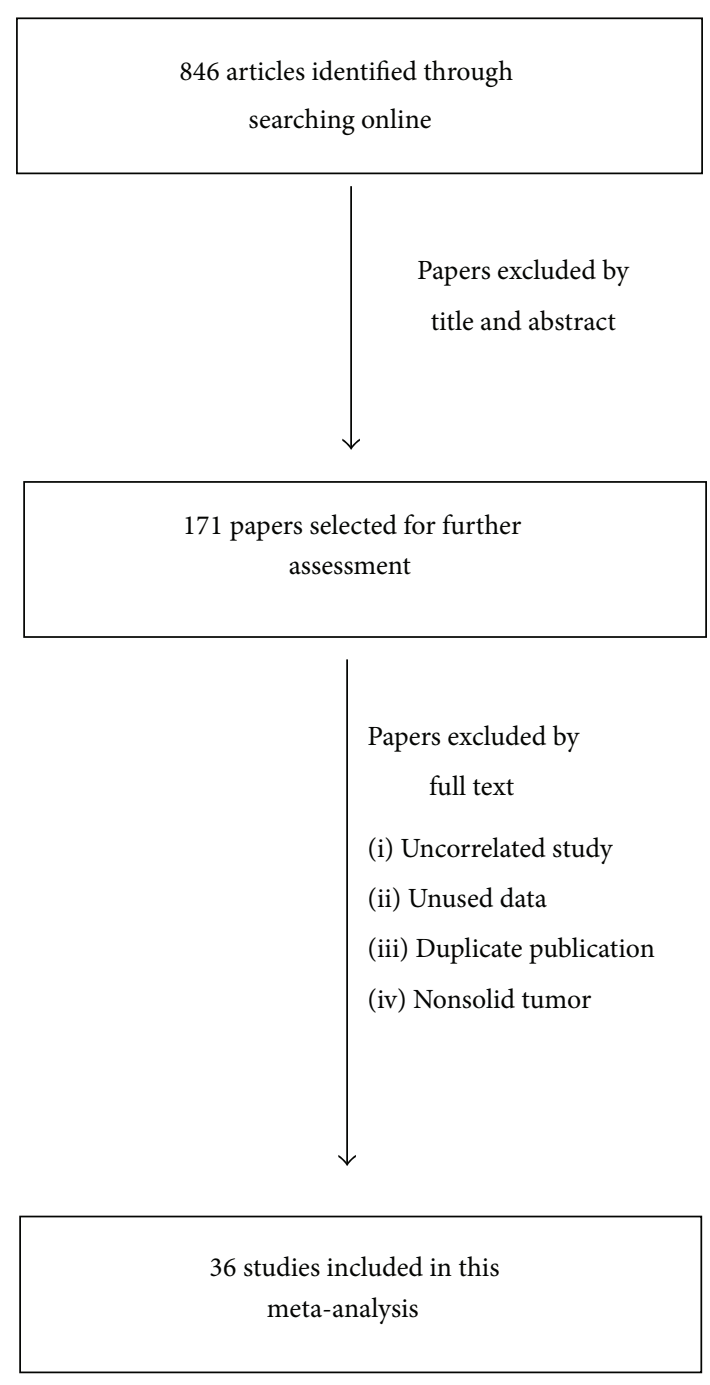

FIGURE 1: Flowchart of study selection in this meta-analysis. 846 studies were preretrieved in accordance with the established search strategies. Then 171 studies that may meet the requirements were further screened out through browsing the titles and abstracts. After reading the full texts of 171 studies, 36 eligible studies were finally included in this meta-analysis according to the criteria.

respectively. In Asian groups, no heterogeneity was found among studies $\left(I^{2}=21.6 \%, P_{h}=0.143\right)$; however, there was a slight heterogeneity in non-Asian groups $\left(I^{2}=47.4 \%, P_{h}=\right.$ $0.107)$. With 100 patients as the boundary, these two groups all prompted that FOXM1 overexpression was not conducive to prognosis $(P<0.001)$, and there was no significant heterogeneity $\left(I^{2}<50 \%\right)$. Moreover, we performed grouping according to the data source of HR (95\% CI) and scores derived from the rating scale we have established, respectively. In both cases, all groups were statistically significant $(P<0.001)$, and no obvious heterogeneity existed among them $\left(I^{2}<50 \%\right)$.

Finally, we divided tumors from the same organ into one group. Four studies related to $\mathrm{BC}$ were divided into the same group, with the combined HR $=2.46(95 \% \mathrm{CI}=1.87-$ 3.22). There was significant statistical significance between the expression of FOXM1 and the prognosis of BC $(P<$ $0.001)$. However, the heterogeneity among various studies was very high $\left(I^{2}=65.1 \%, P_{h}=0.035\right)$. Similar situation occurred in HCC group, with the combined HR $=1.90$ (95\% $\left.\mathrm{CI}=1.48-2.44, P<0.001 ; I^{2}=59.5 \%, P_{h}=0.06\right)$. In GC group, the combined $\mathrm{HR}=2.27(95 \% \mathrm{CI}=1.13-4.58, P=$ $\left.0.022 ; I^{2}=0 \%, P_{h}=0.747\right)$. In PDA group, the combined $\mathrm{HR}=1.73\left(95 \% \mathrm{CI}=1.05-2.86, P=0.032 ; I^{2}=47.2 \%, P_{h}\right.$ $=0.151)$. There were 7 studies in NSCLC group, the combined $\mathrm{HR}=1.82\left(95 \% \mathrm{CI}=1.46-2.28 ; I^{2}=0 \%, P_{h}=0.864\right)$, which showed a significant statistical significance $(P<0.001)$ and no heterogeneity. Surprisingly, the $95 \%$ CI of HR in ovarian cancer showed an overlap with $1(P=0.084)$. In addition, oral cancer, laryngeal SCC, cervical cancer, and esophagus cancer among 36 studies that were included were all identified as SCC. Individual analysis of these studies revealed that FOXM1 also had predictive value in SCC ( $\mathrm{HR}=2.20,95 \%$ $\left.\mathrm{CI}=1.55-3.12, P<0.001 ; I^{2}=64.4 \%, P_{h}=0.038\right)$.

3.3. Publication Bias. Funnel plot analysis showed no publication bias among the 36 studies included (Figure 3). In order to avoid influence of subjective judgment on the conclusion, we validated whether there was publication bias using Begg's test and Egger's test. Indeed, the results revealed no evidence of publication bias $\left(P_{\text {begg }}=0.089, P_{\text {egger }}=0.127\right)$. Furthermore, we also expressed the results by the fixed effect model so as to compare the differences and evaluate the sensitivity of the meta-analyses. The sensitivity analysis showed that no individual study significantly influenced the combined HR, indicating the robust result of this meta-analysis (Figure 4).

\section{Discussion}

Information provided by individual study was limited. To our knowledge, this meta-analysis was the first study to systematically assess the association between FOXM1 expression and the prognostic factors of MST. Our meta-analysis and systematic review showed that the prognosis of patients with tumors overexpressing FOXM1 was significantly worse than patients with low expression of FOXM1.

How FOXM1 affected the prognosis of patients and what role FOXM1 played in MST have gradually become clear. FOXM1 had the common characteristic of Fox transcription factors family: a conserved DNA sequence with winged helix domain [50]. Human FOXM1 gene, which was about $25 \mathrm{~kb}$, was located in 12p13-3 chromosome band (telomere position) and composed of ten exons [51]. FOXM1 was involved in the development and growth of the tumors. The most important function of FOXM1 was to regulate cell cycle, promote cell proliferation, and inhibit cell aging and apoptosis. Cell cycle and its functioning were an orderly process of gene regulation, with the participation of a number of cyclins and cyclin-dependent kinases (Cdks). In quiescent cells, FOXM1 was hardly expressed, while, in cell proliferation, FOXM1 showed high expression. It was involved in the transcription regulation of many genes related to cell cycle and thereby 


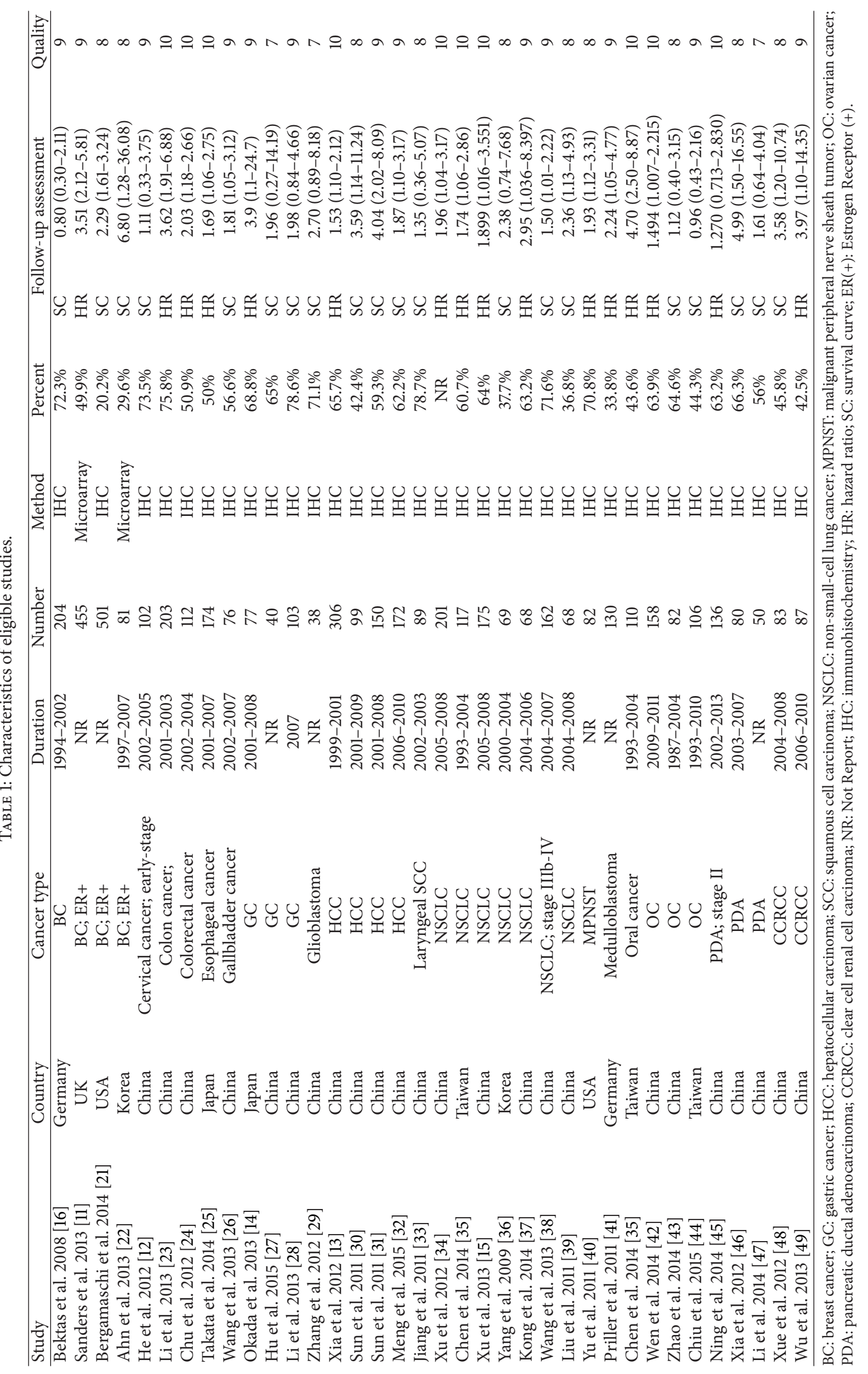




\begin{tabular}{|c|c|c|c|}
\hline Study ID & & HR $(95 \%$ CI $)$ & Weight (\%) \\
\hline Ahn et al. 2013 & $\longrightarrow$ & $6.80(1.28,36.08)$ & 0.39 \\
\hline Bektas et al. 2008 & & $0.80(0.30,2.11)$ & 1.15 \\
\hline Sanders et al. 2013 & & $3.51(2.12,5.81)$ & 4.31 \\
\hline Bergamaschi et al. 2014 & & $2.29(1.61,3.24)$ & 8.96 \\
\hline Okada et al. 2013 & $\longrightarrow$ & $3.90(1.10,24.70)$ & 0.45 \\
\hline Hu et al. 2015 & - & $1.96(0.27,14.19)$ & 0.28 \\
\hline Li et al. 2013 & & $1.98(0.84,4.66)$ & 1.49 \\
\hline Xia et al. 2012 & - & $1.53(1.10,2.12)$ & 10.17 \\
\hline Sun et al. 2011 & 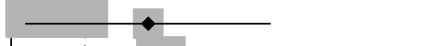 & $3.59(1.14,11.24)$ & 0.84 \\
\hline Sun et al. 2011 & & $4.04(2.02,8.09)$ & 2.27 \\
\hline Meng et al. 2015 & & $1.87(1.10,3.17)$ & 3.91 \\
\hline Xu et al. 2012 & & $1.96(1.04,3.17)$ & 3.53 \\
\hline Chen et al. 2014 & & $1.74(1.06,2.86)$ & 4.45 \\
\hline Xu et al. 2013 & & $1.90(1.02,3.55)$ & 2.80 \\
\hline Yang et al. 2009 & & $2.38(0.74,7.68)$ & 0.80 \\
\hline Kong et al. 2014 & $\bullet$ & $2.95(1.04,8.40)$ & 1.00 \\
\hline Wang et al. 2013 & & $1.50(1.01,2.22)$ & 7.06 \\
\hline Liu et al. 2011 & $\longrightarrow$ & $2.36(1.13,4.93)$ & 2.02 \\
\hline Wen et al. 2014 & & $1.49(1.01,2.21)$ & 7.05 \\
\hline Zhao et al. 2014 & 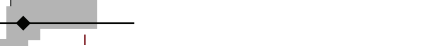 & $1.12(0.40,3.15)$ & 1.03 \\
\hline Chiu et al. 2015 & 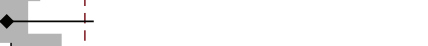 & $0.96(0.43,2.16)$ & 1.68 \\
\hline Ning et al. 2014 & $\rightarrow$ & $1.27(0.71,2.83)$ & 2.30 \\
\hline Xia et al. 2012 & $\rightarrow$ & $4.99(1.50,16.55)$ & 0.76 \\
\hline Li et al. 2014 & $\rightarrow$ & $1.61(0.64,4.04)$ & 1.29 \\
\hline Xue et al. 2012 & $\leftrightarrow$ & $3.58(1.20,10.74)$ & 0.91 \\
\hline Wu et al. 2013 & & $3.97(1.10,14.35)$ & 0.66 \\
\hline He et al. 2012 & $\begin{array}{l}* \\
* \quad 1\end{array}$ & $1.11(0.33,3.75)$ & 0.74 \\
\hline Li et al. 2013 & - & $3.62(1.91,6.88)$ & 2.67 \\
\hline Chu et al. 2012 & $\longrightarrow$ & $2.03(1.18,2.66)$ & 6.63 \\
\hline Takata et al. 2014 & & $1.69(1.06,2.75)$ & 4.82 \\
\hline Wang et al. 2013 & - & $1.81(1.05,3.12)$ & 3.69 \\
\hline Zhang et al. 2012 & +1 & $2.70(0.89,8.18)$ & 0.89 \\
\hline Jiang et al. 2011 & $\rightarrow 1$ & $1.35(0.36,5.07)$ & 0.63 \\
\hline Yu et al. 2011 & $\longrightarrow$ & $1.93(1.12,3.31)$ & 3.73 \\
\hline Priller et al. 2011 & & $2.24(1.05,4.77)$ & 1.91 \\
\hline Chen et al. 2014 & $\bullet$ & $4.70(2.50,8.87)$ & 2.73 \\
\hline Overall $\left(I^{2}=26.4 \%\right.$, & $\diamond$ & $1.99(1.79,2.21)$ & 100.00 \\
\hline 7 & \multirow{2}{*}{\multicolumn{3}{|c|}{$\begin{array}{c} \\
36.1 \\
\end{array}$}} \\
\hline 0.0277 & & & \\
\hline
\end{tabular}

FIGURE 2: Meta-analysis of the association between FOXM1 expression and survival in MST. Combine the data of 36 studies on MST to assess the survival. The results indicated statistically significant difference $(\mathrm{HR}=1.99,95 \% \mathrm{CI}=1.79-2.21$, and $P<0.001)$ and slight between-research heterogeneity $\left(I^{2}=26.4 \%, P_{h}=0.076\right)$ between MST prognosis and FOXM1 expression.

controlled the cellular DNA replication and mitosis process $[52,53]$. Pathological overexpression of FOXM1 can induce the malignant proliferation of tumor cells. The main signaling pathways involved included FOXM1-Skp2-p27 [54, 55], FOXM1-ras-ROS [56, 57], and FOXM1-Raf/MEK/MAPK [58]. In addition, FOXM1 can activate the transcription of C-myc, which was an important signaling molecule to stimulate cell proliferation $[59,60]$. On the other hand, FOXM1 also regulated the expression of Cdc25A phosphatase that was necessary for activating DNA replication; therefore, FOXM1 missing can naturally inhibit mitosis process [53]. FOXM1 was closely related to cell senescence as well. FOXM1 suppressed the expression of p53 and thereby significantly inhibited cellular aging $[61,62]$. In short, FOXM1 maintained the homeostasis between cell proliferation and apoptosis; breaking the balance may promote tumorigenesis [4]. In addition, FOXM1 was also involved in the invasion and metastasis of tumors. It has shown that FOXM1 promoted tumor metastasis by regulating epithelial-mesenchymal conversion (EMT) in tumor cells $[63,64]$. However, some studies demonstrated that FOXM1 promoted tumor metastasis by regulating the expression of MMPs [13, 65-67]. Moreover, FOXM1 also accelerated angiogenesis via upregulation of VEGF expression and thereby promoted tumor metastasis $[68,69]$. All in all, these results suggested that FOXM1 promoted or facilitated the invasion and metastasis of tumor 
TABle 2: Meta-analysis.

\begin{tabular}{|c|c|c|c|c|c|}
\hline & Study & HR (95\% CI) & $P$ & $I^{2}$ & $P_{h}$ \\
\hline \multicolumn{6}{|l|}{ Region } \\
\hline Asian & 31 & $1.92(1.71-2.16)$ & $<0.001$ & $21.6 \%$ & 0.143 \\
\hline Non-Asian & 5 & $2.28(1.81-2.89)$ & $<0.001$ & $47.4 \%$ & 0.107 \\
\hline \multicolumn{6}{|c|}{ Number of patients } \\
\hline$\leq 100$ & 16 & $2.27(1.79-2.88)$ & $<0.001$ & $0 \%$ & 0.814 \\
\hline$>100$ & 20 & $1.93(1.71-2.16)$ & $<0.001$ & $47.2 \%$ & 0.011 \\
\hline \multicolumn{6}{|l|}{ Sources of HR } \\
\hline $\mathrm{HR}$ & 16 & $2.01(1.75-2.30)$ & $<0.001$ & $39.2 \%$ & 0.055 \\
\hline $\mathrm{SC}$ & 20 & $1.96(1.67-2.31)$ & $<0.001$ & $17 \%$ & 0.242 \\
\hline \multicolumn{6}{|l|}{ Quality score } \\
\hline $5-8$ & 13 & $2.27(1.82-2.83)$ & $<0.001$ & $0 \%$ & 0.785 \\
\hline $9-10$ & 23 & $1.91(1.70-2.15)$ & $<0.001$ & $41.7 \%$ & 0.020 \\
\hline \multicolumn{6}{|l|}{ Cancer type } \\
\hline $\mathrm{BC}$ & 4 & $2.46(1.87-3.22)$ & $<0.001$ & $65.1 \%$ & 0.035 \\
\hline GC & 3 & $2.27(1.13-4.58)$ & 0.022 & $0 \%$ & 0.747 \\
\hline HCC & 4 & $1.90(1.48-2.44)$ & $<0.001$ & $59.5 \%$ & 0.060 \\
\hline NSCLC & 7 & $1.82(1.46-2.28)$ & $<0.001$ & $0 \%$ & 0.864 \\
\hline PDA & 3 & $1.73(1.05-2.86)$ & 0.032 & $47.2 \%$ & 0.151 \\
\hline $\mathrm{OC}$ & 3 & $1.34(0.96-1.88)$ & 0.084 & $0 \%$ & 0.587 \\
\hline Others & 12 & $2.04(1.72-2.42)$ & $<0.001$ & $27.6 \%$ & 0.159 \\
\hline Overall & 36 & $2.02(1.81-2.25)$ & $<0.001$ & $25.6 \%$ & 0.09 \\
\hline
\end{tabular}

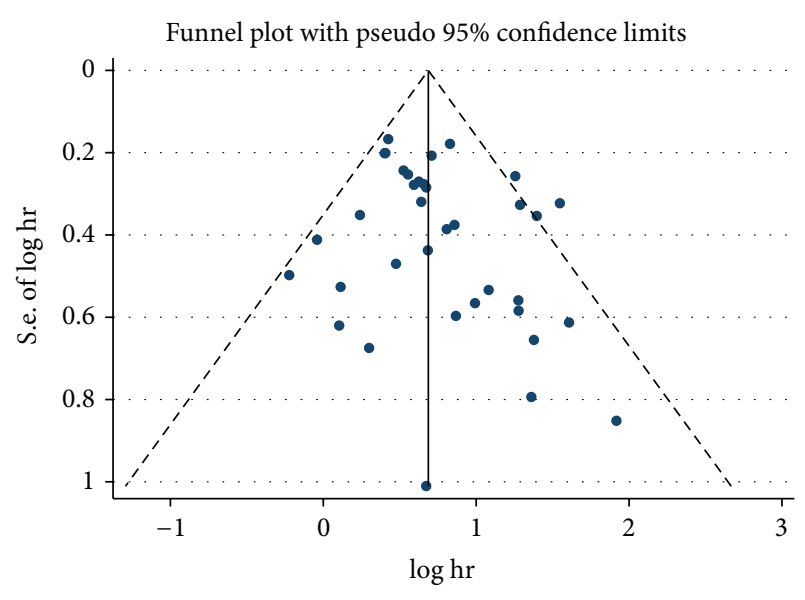

FIGURE 3: Funnel plots for study of MST patients. Funnel plot analysis showed no publication bias among the 36 studies included.

cells through a variety of pathways. In summary, FOXM1 was involved in tumor proliferation, invasion, metastasis, and angiogenesis by regulating the expression of downstream genes related to the tumor and thereby affected the prognosis of patients.

Our research revealed that FOXM1 played a potentially important role in MST; however, we should be extra cautious when applying these results into clinical practice. As many types of tumors were contained in 36 included studies, the biological characteristics of the tumors were various. To further investigate the predictive value of FOXM1 in different types of tumors, we analyzed the combined HR of different groups. In $\mathrm{BC}$ group, we obtained meaningful results after combining the HR of 4 studies; however, the heterogeneity was great $\left(I^{2}>50 \%\right)$. Among these 4 studies, one study was targeted for breast cancer of all types, while the other three opted for ER-positive BC. After excluding this study, we got more accredited results $(\mathrm{HR}=2.70,95 \% \mathrm{CI}=2.04-3.59$, $\left.P<0.001 ; I^{2}=34.8 \%, P_{h}=0.216\right)$. As a consequence, we were more inclined to believe that FOXM1 had a reliable predictive significance in ER-positive BC patients; nonetheless, this did not mean that FOXM1 did not have predictive value in $\mathrm{BC}$ of all types. More study will be able to make up for the vacancy in the future. In GC, PDA, and HCC group, overexpression of FOXM1 all prompted worse prognosis in patients. In GC group, 95\% CI overlapped with " 1 " in some studies [27, 28]. Likewise, in PDA group, 95\% CI in some studies also overlapped with "1" $[45,47]$. Even when there were only 3 studies combined, the results were still meaningful. In HCC group, although it had significant statistical significance, its heterogeneity was very obvious. In NSCLC group, 7 studies were included. The conclusion that overexpression of FOXM1 prompted worse prognosis in patients also applied to NSCLC, but it was more stable and reliable $\left(I^{2}=0 \%\right)$.

Publication bias was a problem to be analyzed in all of the meta-analysis. In this study, we found no publication bias by Begg's test and Egger's test. Funnel plot also revealed no obvious bias. However, some studies had not been included because they did not meet our screening conditions. If we can get the raw data from these studies, reanalysis will be needed. In addition, although analyzing the time-to-event outcomes via the HR had very distinct advantages, there was also a problem. Since follow-up time of each study was not the same, 


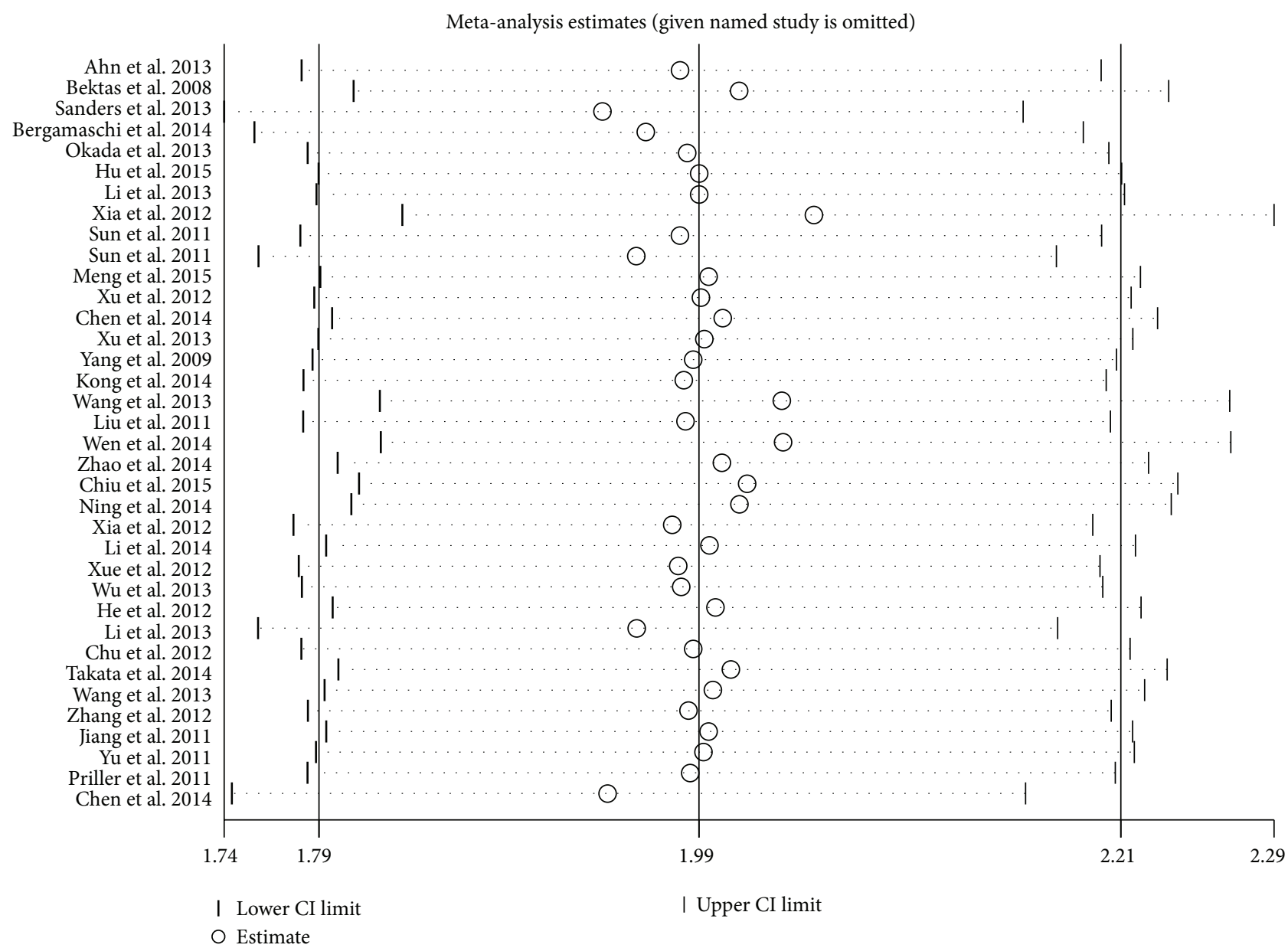

FIGURE 4: Sensitivity analysis. The sensitivity analysis showed that no individual study significantly influenced the combined HR, indicating the robust result of this meta-analysis.

heterogeneity was virtually brought in. Due to the limitation of statistical methods, we should be particularly cautious in the interpretation of the results.

In conclusion, our research suggested that overexpression of FOXM1 may lead to poor prognosis. With the gradual deepening of the study, FOXM1 may become an important target for cancer therapy in the future.

\section{Conflict of Interests}

The authors declare that they have no conflict of interests in relation to the work described.

\section{Authors' Contribution}

Jun Dai and Lili Yang contributed equally to this work.

\section{Acknowledgment}

This work was supported by grants from Natural Science Foundation of China (no. 30570725).

\section{References}

[1] A. Jemal, F. Bray, M. M. Center, J. Ferlay, E. Ward, and D. Forman, "Global cancer statistics," CA: Cancer Journal for Clinicians, vol. 61, no. 2, pp. 69-90, 2011.

[2] M. M. Kirstein, A. Lange, A. Prenzler, M. P. M. Anns, S. Kubicka, and A. Vogel, "Targeted therapies in metastatic colorectal cancer: a systematic review and assessment of currently available data," Oncologist, vol. 19, no. 11, pp. 1156-1168, 2014.

[3] M. S. Anscher, "Targeting the TGF- $\beta 1$ pathway to prevent normal tissue injury after cancer therapy," The Oncologist, vol. 15, no. 4, pp. 350-359, 2010.

[4] C. Pilarsky, M. Wenzig, T. Specht, H. D. Saeger, and R. Grützmann, "Identification and validation of commonly overexpressed genes in solid tumors by comparison of microarray data," Neoplasia, vol. 6, no. 6, pp. 744-750, 2004.

[5] Z. Wang, A. Ahmad, Y. Li, S. Banerjee, D. Kong, and F. H. Sarkar, "Forkhead box M1 transcription factor: a novel target for cancer therapy," Cancer Treatment Reviews, vol. 36, no. 2, pp. 151-156, 2010.

[6] I. Wierstra and J. Alves, "FOXM1, a typical proliferationassociated transcription factor," Biological Chemistry, vol. 388, no. 12, pp. 1257-1274, 2007. 
[7] J. Laoukili, M. R. H. Kooistra, A. Brás et al., "FoxM1 is required for execution of the mitotic programme and chromosome stability," Nature Cell Biology, vol. 7, no. 2, pp. 126-136, 2005.

[8] J. Laoukili, M. Stahl, and R. H. Medema, "FoxM1: at the crossroads of ageing and cancer," Biochimica et Biophysica Acta, vol. 1775, no. 1, pp. 92-102, 2007.

[9] P. Raychaudhuri and H. J. Park, "FoxM1: a master regulator of tumor metastasis," Cancer Research, vol. 71, no. 13, pp. 43294333, 2011.

[10] E. Gemenetzidis, A. Bose, A. M. Riaz et al., "FOXM1 upregulation is an early event in human squamous cell carcinoma and it is enhanced by nicotine during malignant transformation," PLoS ONE, vol. 4, no. 3, Article ID e4849, 2009.

[11] D. A. Sanders, C. S. Ross-Innes, D. Beraldi, J. S. Carroll, and S. Balasubramanian, "Genome-wide mapping of FOXM1 binding reveals co-binding with estrogen receptor alpha in breast cancer cells," Genome Biology, vol. 14, article R6, 2013.

[12] S.-Y. He, H.-W. Shen, L. Xu et al., "FOXM1 promotes tumor cell invasion and correlates with poor prognosis in early-stage cervical cancer," Gynecologic Oncology, vol. 127, no. 3, pp. 601610, 2012.

[13] L. Xia, W. Huang, D. Tian et al., "Upregulated FoxM1 expression induced by hepatitis B virus X protein promotes tumor metastasis and indicates poor prognosis in hepatitis $\mathrm{B}$ virus-related hepatocellular carcinoma," Journal of Hepatology, vol. 57, no. 3 , pp. 600-612, 2012.

[14] K. Okada, Y. Fujiwara, T. Takahashi et al., "Overexpression of forkhead box M1 transcription factor (FOXM1) is a potential prognostic marker and enhances chemoresistance for docetaxel in gastric cancer," Annals of Surgical Oncology, vol. 20, no. 3, pp. 1035-1043, 2013.

[15] N. Xu, D. Jia, W. Chen et al., "FoxM1 is associated with poor prognosis of non-small cell lung cancer patients through promoting tumor metastasis," PLoS ONE, vol. 8, no. 3, Article ID e59412, 2013.

[16] N. Bektas, A. ten Haaf, J. Veeck et al., “Tight correlation between expression of the Forkhead transcription factor FOXM1 and HER2 in human breast cancer," BMC Cancer, vol. 8, article 42, 2008.

[17] M. K. B. Parmar, V. Torri, and L. Stewart, "Extracting summary statistics to perform meta-analyses of the published literature for survival endpoints," Statistics in Medicine, vol. 17, no. 24, pp. 2815-2834, 1998.

[18] J. F. Tierney, L. A. Stewart, D. Ghersi, S. Burdett, and M. R. Sydes, "Practical methods for incorporating summary time-toevent data into meta-analysis," Trials, vol. 8, article 16, 2007.

[19] J. P. T. Higgins, S. G. Thompson, J. J. Deeks, and D. G. Altman, "Measuring inconsistency in meta-analyses," British Medical Journal, vol. 327, no. 7414, pp. 557-560, 2003.

[20] N. Geller, L. Freedman, Y. J. Lee, and R. DerSimonian, “Conference on meta-analysis in the design and monitoring of clinical trials," Statistics in Medicine, vol. 18, no. 6, pp. 753-754, 1999.

[21] A. Bergamaschi, Z. Madak-Erdogan, Y. Kim, Y. Choi, H. Lu, and B. S. Katzenellenbogen, "The forkhead transcription factor FOXM1 promotes endocrine resistance and invasiveness in estrogen receptor-positive breast cancer by expansion of stemlike cancer cells," Breast Cancer Research, vol. 16, no. 5, article 436, 2014

[22] S. G. Ahn, H. M. Lee, H. W. Lee et al., "Prognostic discrimination using a 70-gene signature among patients with estrogen receptor-positive breast cancer and an intermediate 21-gene recurrence score," International Journal of Molecular Sciences, vol. 14, no. 12, pp. 23685-23699, 2013.

[23] D. Li, P. Wei, Z. Peng et al., "The critical role of dysregulated FOXM1-PLAUR signaling in human colon cancer progression and metastasis," Clinical Cancer Research, vol. 19, no. 1, pp. 6272, 2013.

[24] X.-Y. Chu, Z.-M. Zhu, L.-B. Chen et al., "FOXM1 expression correlates with tumor invasion and a poor prognosis of colorectal cancer," Acta Histochemica, vol. 114, no. 8, pp. 755-762, 2012.

[25] A. Takata, S. Takiguchi, K. Okada et al., "Clinicopathological and prognostic significance of FOXM1 expression in esophageal squamous cell carcinoma," Anticancer Research, vol. 34, no. 5, pp. 2427-2432, 2014.

[26] R. Wang, Y. Song, X. Xu, Q. Wu, and C. Liu, "The expression of Nek7, FoxM1, and Plk1 in gallbladder cancer and their relationships to clinicopathologic features and survival," Clinical and Translational Oncology, vol. 15, no. 8, pp. 626-632, 2013.

[27] C.-J. Hu, B. Wang, B. Tang et al., "The FOXM1-induced resistance to oxaliplatin is partially mediated by its novel target gene Mcl-1 in gastric cancer cells," Biochimica et Biophysica Acta-Gene Regulatory Mechanisms, vol. 1849, no. 3, pp. 290299, 2015.

[28] X. Li, W. Qiu, B. Liu et al., "Forkhead box transcription factor 1 expression in gastric cancer: FOXM1 is a poor prognostic factor and mediates resistance to docetaxel," Journal of Translational Medicine, vol. 11, article 204, 2013.

[29] N. Zhang, X. Wu, L. Yang et al., "FoxM1 inhibition sensitizes resistant glioblastoma cells to temozolomide by downregulating the expression of DNA-repair gene Rad51," Clinical Cancer Research, vol. 18, no. 21, pp. 5961-5971, 2012.

[30] H. Sun, M. Teng, J. Liu et al., "FOXM1 expression predicts the prognosis in hepatocellular carcinoma patients after orthotopic liver transplantation combined with the Milan criteria," Cancer Letters, vol. 306, no. 2, pp. 214-222, 2011.

[31] H.-C. Sun, M. Li, J.-L. Lu et al., "Overexpression of Forkhead box M1 protein associates with aggressive tumor features and poor prognosis of hepatocellular carcinoma," Oncology Reports, vol. 25, no. 6, pp. 1533-1539, 2011.

[32] F.-D. Meng, J.-C. Wei, K. Qu et al., "FoxM1 overexpression promotes epithelial-mesenchymal transition and metastasis of hepatocellular carcinoma," World Journal of Gastroenterology, vol. 21, no. 1, pp. 196-213, 2015.

[33] L.-Z. Jiang, P. Wang, B. Deng et al., "Overexpression of Forkhead Box M1 transcription factor and nuclear factor- $\kappa \mathrm{B}$ in laryngeal squamous cell carcinoma: a potential indicator for poor prognosis," Human Pathology, vol. 42, no. 8, pp. 1185-1193, 2011.

[34] N. Xu, S.-D. Wu, H. Wang, Q. Wang, and C.-X. Bai, "Involvement of foxml in non-small cell lung cancer recurrence," Asian Pacific Journal of Cancer Prevention, vol. 13, no. 9, pp. 47394743, 2012.

[35] P. Chen, Y. Cheng, Y. Wang, T. Wu, C. Chen, and H. Lee, "Up-regulation of FOXM1 by E6 oncoprotein through the MZF1/NKX2-1 axis is required for human papillomavirusassociated tumorigenesis," Neoplasia, vol. 16, no. 11, pp. 961-971, 2014.

[36] D. K. Yang, C. H. Son, S. K. Lee, P. J. Choi, K. E. Lee, and M. S. Roh, "Forkhead box M1 expression in pulmonary squamous cell carcinoma: correlation with clinicopathologic features and its prognostic significance," Human Pathology, vol. 40, no. 4, pp. 464-470, 2009. 
[37] F.-F. Kong, Z.-Q. Qu, H.-H. Yuan et al., "Overexpression of FOXM1 is associated with EMT and is a predictor of poor prognosis in non-small cell lung cancer," Oncology Reports, vol. 31, no. 6, pp. 2660-2668, 2014.

[38] Y. Wang, L. Wen, S.-H. Zhao, Z.-H. Ai, J.-Z. Guo, and W.-C. Liu, "FoxM1 expression is significantly associated with cisplatinbased chemotherapy resistance and poor prognosis in advanced non-small cell lung cancer patients," Lung Cancer, vol. 79, no. 2, pp. 173-179, 2013.

[39] Y.-Q. Liu, R.-H. Guo, L.-K. Liu et al., "Correlation between expression of forkhead box M1 (FOXM1) and clinicopathological features and prognosis in patients with non-small cell lung cancer (NSCLC)," Zhonghua Zhong Liu Za Zhi, vol. 33, no. 6, pp. 426-430, 2011.

[40] J. Yu, H. Deshmukh, J. E. Payton et al., "Array-based comparative genomic hybridization identifies CDK4 and FOXM1 alterations as independent predictors of survival in malignant peripheral nerve sheath tumor," Clinical Cancer Research, vol. 17, no. 7, pp. 1924-1934, 2011.

[41] M. Priller, J. Pöschl, L. Abrão et al., "Expression of FoxM1 is required for the proliferation of medulloblastoma cells and indicates worse survival of patients," Clinical Cancer Research, vol. 17, no. 21, pp. 6791-6801, 2011.

[42] N. Wen, Y. Wang, L. Wen et al., "Overexpression of FOXM1 predicts poor prognosis and promotes cancer cell proliferation, migration and invasion in epithelial ovarian cancer," Journal of Translational Medicine, vol. 12, article 134, 2014.

[43] F. Zhao, M. K. Y. Siu, L. Jiang et al., "Overexpression of forkhead box protein M1 (FOXM1) in ovarian cancer correlates with poor patient survival and contributes to paclitaxel resistance," PLoS ONE, vol. 9, no. 11, Article ID el13478, 2014.

[44] W.-T. Chiu, Y.-F. Huang, H.-Y. Tsai et al., "FOXM1 confers to epithelial-mesenchymal transition, stemness and chemoresistance in epithelial ovarian carcinoma cells," Oncotarget, vol. 6, no. 4, pp. 2349-2365, 2015.

[45] Z. Ning, A. Wang, J. Liang et al., "USP22 promotes the G1/S phase transition by upregulating FoxM1 expression via $\beta$ catenin nuclear localization and is associated with poor prognosis in stage II pancreatic ductal adenocarcinoma," International Journal of Oncology, vol. 45, no. 4, pp. 1594-1608, 2014.

[46] J.-T. Xia, H. Wang, L.-J. Liang et al., "Overexpression of FOXM1 is associated with poor prognosis and clinicopathologic stage of pancreatic ductal adenocarcinoma," Pancreas, vol. 41, no. 4, pp. 629-635, 2012.

[47] L. Li, Z. Li, X. Kong et al., "Down-regulation of MicroRNA-494 via loss of SMAD4 increases FOXM1 and $\beta$-catenin signaling in pancreatic ductal adenocarcinoma cells," Gastroenterology, vol. 147, no. 2, pp. 485-497, 2014.

[48] Y.-J. Xue, R.-H. Xiao, D.-Z. Long et al., "Overexpression of FoxM1 is associated with tumor progression in patients with clear cell renal cell carcinoma," Journal of Translational Medicine, vol. 10, no. 1, article 200, 2012.

[49] X.-R. Wu, Y.-H. Chen, D.-M. Liu et al., "Increased expression of forkhead box M1 protein is associated with poor prognosis in clear cell renal cell carcinoma," Medical Oncology, vol. 30, no. 1, article 346, 2013.

[50] H. Ye, T. F. Kelly, U. Samadani et al., "Hepatocyte nuclear factor $3 /$ fork head homolog 11 is expressed in proliferating epithelial and mesenchymal cells of embryonic and adult tissues," Molecular and Cellular Biology, vol. 17, no. 3, pp. 1626-1641, 1997.

[51] W. Korver, J. Roose, K. Heinen et al., "The human TRIDENT/HFH-11/FKHL16 gene: structure, localization, and promoter characterization," Genomics, vol. 46, no. 3, pp. 435-442, 1997.

[52] I.-M. Kim, T. Ackerson, S. Ramakrishna et al., "The Forkhead Box $\mathrm{ml}$ transcription factor stimulates the proliferation of tumor cells during development of lung cancer," Cancer Research, vol. 66, no. 4, pp. 2153-2161, 2006.

[53] Y. Yoshida, I.-C. Wang, H. M. Yoder, N. O. Davidson, and R. H. Costa, "The forkhead box M1 transcription factor contributes to the development and growth of mouse colorectal cancer," Gastroenterology, vol. 132, no. 4, pp. 1420-1431, 2007.

[54] M. Liu, B. Dai, S.-H. Kang et al., "FoxM1B is overexpressed in human glioblastomas and critically regulates the tumorigenicity of glioma cells," Cancer Research, vol. 66, no. 7, pp. 3593-3602, 2006.

[55] V. Petrovic, R. H. Costa, L. F. Lau, P. Raychaudhuri, and A. L. Tyner, "FoxM1 regulates growth factor-induced expression of kinase-interacting stathmin (KIS) to promote cell cycle progression," Journal of Biological Chemistry, vol. 283, no. 1, pp. 453-460, 2008.

[56] H. J. Park, R. H. Costa, L. F. Lau, A. L. Tyner, and P. Raychaudhuri, "Anaphase-promoting complex/cyclosome-Cdh1mediated proteolysis of the forkhead box M1 transcription factor is critical for regulated entry into S phase," Molecular and Cellular Biology, vol. 28, no. 17, pp. 5162-5171, 2008.

[57] C. G. Havens, A. Ho, N. Yoshioka, and S. F. Dowdy, "Regulation of late G1/S phase transition and APCCdh1 by reactive oxygen species," Molecular and Cellular Biology, vol. 26, no. 12, pp. 47014711, 2006.

[58] R. Y. M. Ma, T. H. K. Tong, A. M. S. Cheung, A. C. C. Tsang, W. Y. Leung, and K.-M. Yao, "Raf/MEK/MAPK signaling stimulates the nuclear translocation and transactivating activity of FOXM1c," Journal of Cell Science, vol. 118, no. 4, pp. 795-806, 2005.

[59] J. Zeng, L. Wang, Q. Li et al., "FoxM1 is up-regulated in gastric cancer and its inhibition leads to cellular senescence, partially dependent on $\mathrm{p} 27^{\text {kipl }}$," Journal of Pathology, vol. 218, no. 4, pp. 419-427, 2009.

[60] I. Wierstra and J. Alves, "FOXM1c transactivates the human cmyc promoter directly via the two TATA boxes P1 and P2," FEBS Journal, vol. 273, no. 20, pp. 4645-4667, 2006.

[61] Y. Tan, P. Raychaudhuri, and R. H. Costa, "Chk2 mediates stabilization of the FoxM1 transcription factor to stimulate expression of DNA repair genes," Molecular and Cellular Biology, vol. 27, no. 3, pp. 1007-1016, 2007.

[62] S. K. Li, D. K. Smith, W. Y. Leung et al., "FoxM1c counteracts oxidative stress-induced senescence and stimulates Bmi-1 expression," The Journal of Biological Chemistry, vol. 283, no. 24, pp. 16545-16553, 2008.

[63] B. Bao, Z. Wang, S. Ali et al., "Over-expression of FoxM1 leads to epithelial-mesenchymal transition and cancer stem cell phenotype in pancreatic cancer cells," Journal of Cellular Biochemistry, vol. 112, no. 9, pp. 2296-2306, 2011.

[64] J. Li, Y. Wang, J. Luo et al., "miR-134 inhibits epithelial to mesenchymal transition by targeting FOXM1 in non-small cell lung cancer cells," FEBS Letters, vol. 586, no. 20, pp. 3761-3765, 2012.

[65] X. Wang, D. Bhattacharyya, M. B. Dennewitz et al., "Rapid hepatocyte nuclear translocation of the Forkhead Box M1B (FoxM1B) transcription factor caused a transient increase in size of regenerating transgenic hepatocytes," Gene Expression, vol. 11, no. 3-4, pp. 149-162, 2003. 
[66] B. Dai, S.-H. Kang, W. Gong et al., "Aberrant FoxM1B expression increases matrix metalloproteinase-2 transcription and enhances the invasion of glioma cells," Oncogene, vol. 26, no. 42, pp. 6212-6219, 2007.

[67] A. Ahmad, Z. Wang, D. Kong et al., "FoxM1 down-regulation leads to inhibition of proliferation, migration and invasion of breast cancer cells through the modulation of extra-cellular matrix degrading factors," Breast Cancer Research and Treatment, vol. 122, no. 2, pp. 337-346, 2010.

[68] Y. Zhang, N. Zhang, B. Dai et al., "FoxM1B transcriptionally regulates vascular endothelial growth factor expression and promotes the angiogenesis and growth of glioma cells," Cancer Research, vol. 68, no. 21, pp. 8733-8742, 2008.

[69] Q. Li, N. Zhang, Z. Jia et al., "Critical role and regulation of transcription factor foxml in human gastric cancer angiogenesis and progression," Cancer Research, vol. 69, no. 8, pp. 35013509, 2009. 


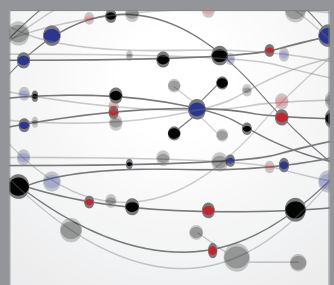

The Scientific World Journal
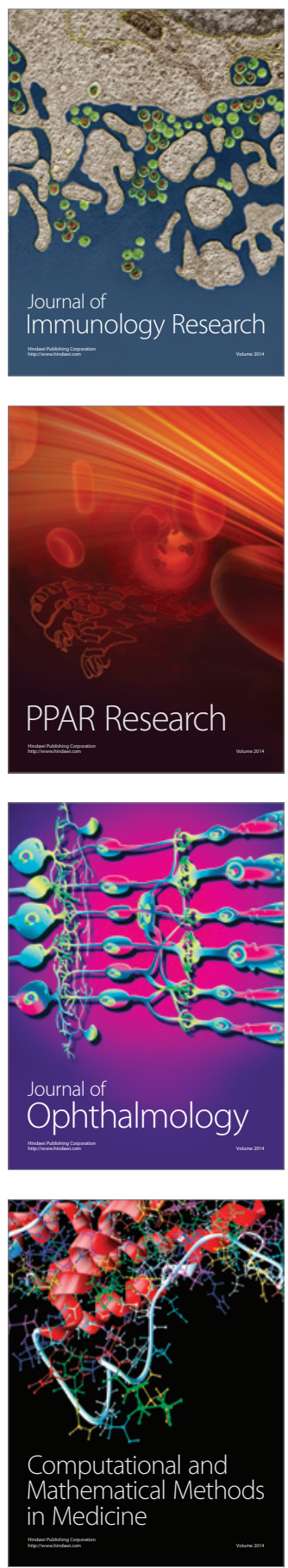

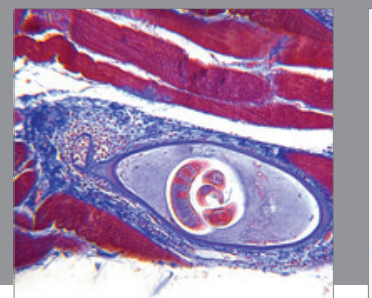

Gastroenterology

Research and Practice
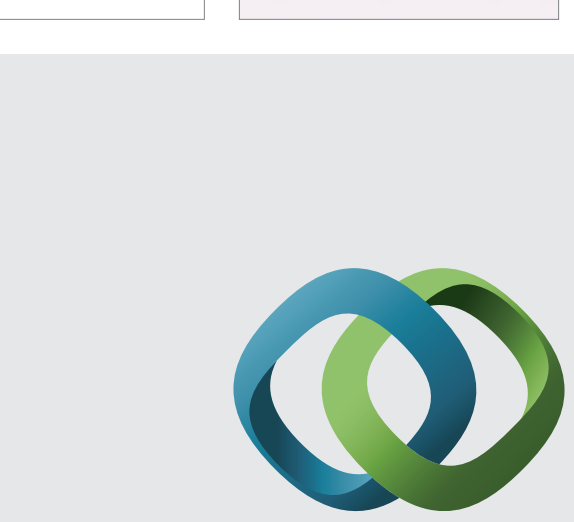

\section{Hindawi}

Submit your manuscripts at

http://www.hindawi.com
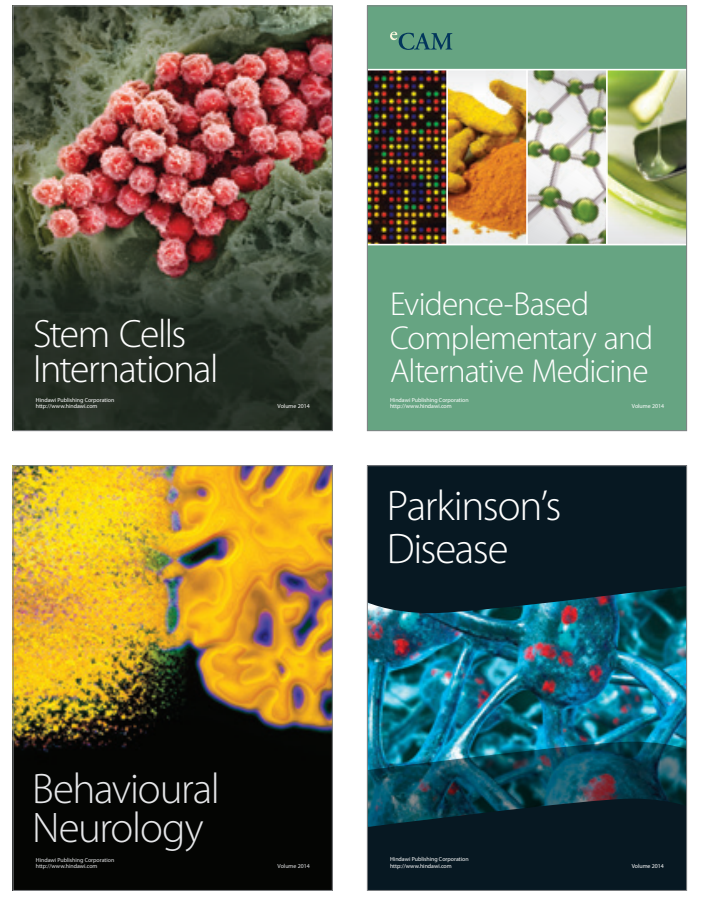
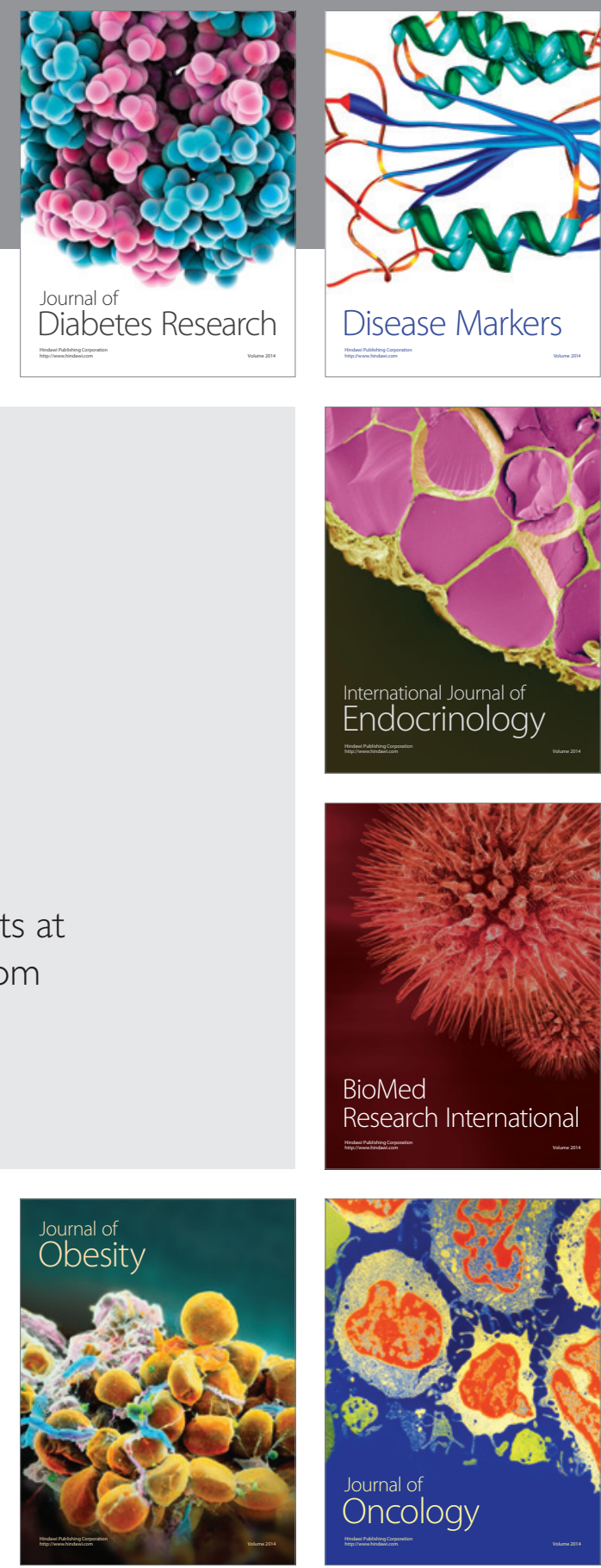

Disease Markers
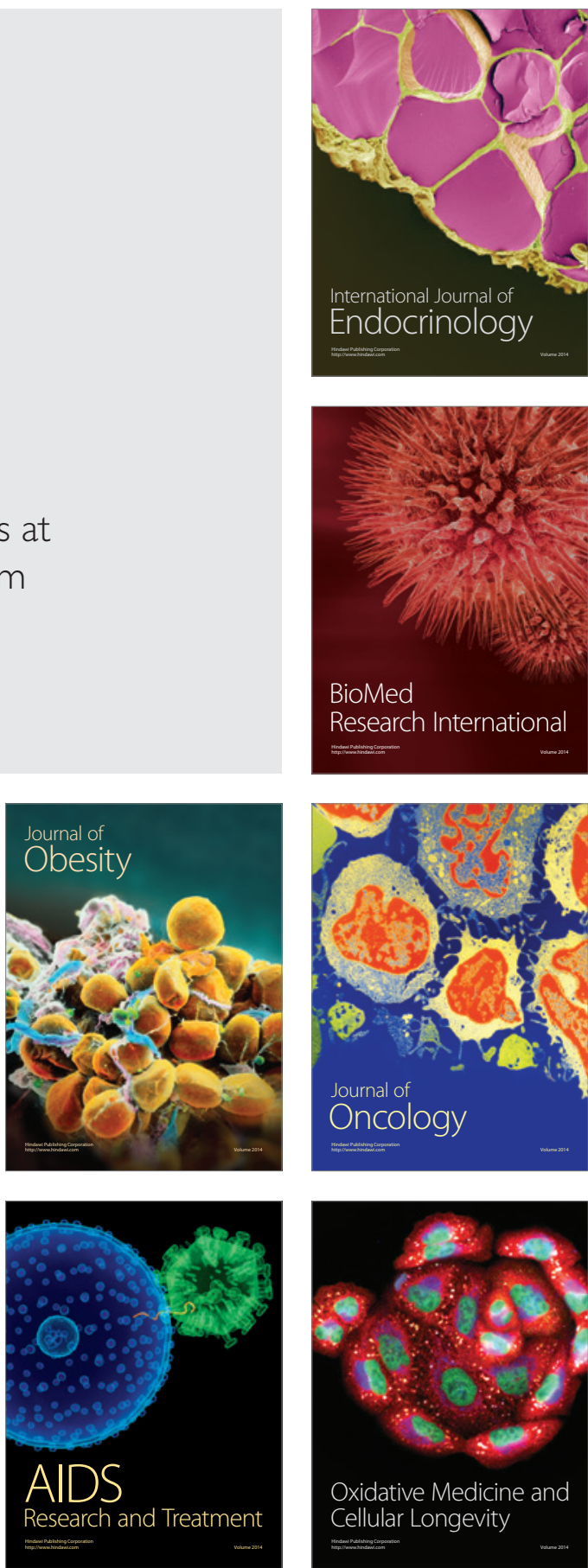